\title{
Méthode chimique de dilution Procédé par intégration
}

\author{
Chemical flow gauging \\ The integration method
}

par Henri ANDRÉ

INGÉNIEUA A LA DIVISION TEGHNIQUE GÉNÉRALE

DU SERVICE DE LA PRODUCTION HYDRAULIQUE DE J'ÉLECTRICITÉ DE FRANCE

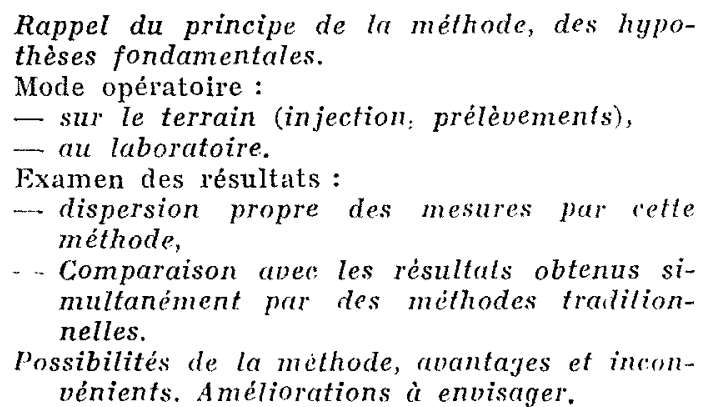
thèses fondamentales.

Mode opératoire :

- au laboratoire.

Examen des résultats:

Comparaison avec les résultats obtenus simultanément par des mithodes tradilionssibilités de la methode, avantajes et
vénients. Améliorations à envisager.

\author{
Review of principles and basic assumptions of \\ the method. \\ Operational procedure: \\ (i) In the field (injection, sample taling), \\ (ii) In the laboratory. \\ Examination of resulls. \\ (i) Inherent measurement error of the method, \\ (ii) Comparison with results obtained at the \\ same time by other methods. \\ The possibilities, advantages and drawbaclis \\ of the methods.-. Suggested improvements.
}

\section{HISTORIQUE SUCCINCT DES MÉTHODES CHIMIQUES DE DILUTION POUR LE JAUGEAGE DES RIVIERES}

La première mesure de débit par dilution semble avoir vu le jour en 1863 sous l'impulsjon du physicien français Th. Schloesing.

Divers sel̄s ont été successivement utilisés, du chlorure de sodium au bichromate de soude en passant par le nitrite, le sulfate de manganèse et la fluorescéine.

Les procédés d'analyse sont diver's : par titration chimique et par analyse colorimétrique depuis 1896 (Stromeyer) ou par mesures de résistivité (W.D. Peaslee en 1915 et Ott en 1921).

Le procédé conduisait en général à étudier la concentration maxinum correspondant au ré- gime permanent établi lors d'une longue injection à débit constant.

En 1952, H. Dumas et M. Dodero, avec la collaboration de la Région d'Equipement Alpes-II d'Electricité de France, ont amélioré considérablement les méthodes utilisées jusque-là en adaptant le matériel aux exigences industrielles des mesures de débits des torrents de haute montagne. L'analyse est conduite par colorimétrie en adoptant une méthode de zéro pour accroître la précision. Des centaines de jaugeages sont réalisés chaque année en France par la méthode de H. Dumas et M. Dodero et donnent des résultats Ires satisfaisants. La Division Technique Géné- 
rale a effectué en 1959 plus de 350 jaugeages par cette méthode, près de 500 en 1960 , et a jaugé avec succès en particulier un débit voisin de $200 \mathrm{~m}^{3} / \mathrm{s}$.

$$
\text { 蒋 }
$$

A. Barbagelata et E. Bottani en 1927, puis Pisa en 1935, eurent l'idée d'intégrer le poids total de sel injecté, en prélevant en tous les points de la section de prélèvement.

L'utilisation de radio-isotopes depuis 1957 permit à Hull de remarquer que le résultat obtenu semblait, dans une large mesure, indépendant de la position du compteur dans la section de pré- lèvement, même en l'absence de tout palier de régime permanent.

Ce résultat nous a paru intéressant et susceptible de permettre l'amélioration et l'allégement du procédé par intégration.

Parallèlement avec le Service des Etudes et Recherches Hydrauliques d'Electricité de France, qui étudie depuis 1959 les possibilités de mesures de débits dans les usines hydrauliques par ce procédé, la Division Technique Générale a entrepris la mise en cuvre, pour les jaugeages de torrents, avec utilisation du bichromate de soude, de ce procédé, qualifié dans les pays anglo-saxons de «Gulp Method» ou de «Total Count Method».

\section{MÉTHODE CHIMIQUE DE DILUTION AU BICHROMATE PROCÉDE PAR INTÉGRATION}

\section{I. - PRINCIPE DE LA MÉTHODE - CONDITIONS FONDAMENTALES}

\section{1-1. - Rappel du principe de la méthode tradi- tionnelle de dilution utilisée en France depuis 1952 (La Honille Blanche, $\mathrm{n}^{\circ} 5$ de 1952 et $n^{\circ} 3$ de 1953 ).}

La méthode traditionnelle de dilution consiste à injecter dans la rivière un débit constant et parfaitement connu $q$ d'une solution concentrée de bichromate à la concentration $C_{1}$ pendant un temps suffisant pour obtenir un palier de concentration $\mathrm{C}_{2}$ dans la rivière au point de prélèvement.

La section de prélèvement est assez loin en aval du lieu d'injection pour que la concentralion de la rivière soit la même en tous les points de la section à tout instant $t$ du palier de concentration (régime permanent).

En supposant que les eaux de la rivière ne contiennent pas de bichromate de soude, ce qui est le cas général, le débit de la rivière est égal à :

$$
\mathrm{Q}=q\left(\mathrm{C}_{1} / \mathrm{C}_{2}\right)-q \# q\left(\mathrm{C}_{1} / \mathrm{C}_{2}\right)
$$

\section{1-2. - Principe de la méthode par intégration proposée.}

Un volume connu $\mathrm{V}$ d'une solutica de bichromate de soude, à la concentration $\mathrm{C}_{1}$, est injecté de façon quelconque dans la rivière.

Des prélèvements sont effectués pendant tout le temps de passage $T$ du bichromate en un point de la rivière situé assez loin à l'aval pour tịe le mélange soit parfaitement réparti dans la section de prélèvement.

Si $\mathrm{C}_{2}$ est la concentration moyenne des $n$ échantillons prélevés pendant le temps ' $T$, la loi de conservation de la matière permet d'écrire ce que nous appelons l'équation fondamentale :

$$
\mathrm{VC}_{1}=\mathrm{QTC}_{2}=\mathrm{QT} \int_{11}^{\mathrm{T}} c_{2} d t / \mathrm{T}
$$

donc :

$$
\mathrm{Q}=\frac{\mathrm{VC}_{1}}{\int_{0}^{\mathrm{T}} c_{2} d t}
$$

\section{I-3. - Conditions fondamentales.}

\section{I-3.1 Première condtTion :}

L'équation (1) n'est valable que si le terme $\int_{0}^{\mathrm{T}} c_{2} d t$ est le même pour tous les points de la section de prélèvement.

Le procédé d'injection étant quelconque, il est évident, en raison de l'hétérogénéité des vitesses, qu'à un instant donné, même si le brassage est parfait, la concentration n'est pas la même en tous les points de la section de prélèvement.

Dans la méthode traditionnelle, l'efficacité du brassage est concrétisée par le fait que la concentration est la même en tous les points de la section de prélèvement pendant la durée du régime permanent. 
Dans la méthode proposée, le brassage est réputé bon dans la section de prélèvement si le terme $\int_{0}^{\mathrm{T}} c_{2} d t$ est constant quel que soit le point de prélèvement choisi dans cette section. I-3.2 DEuxième conditron : Conservation de la matière.
Dans la méthode traditionnelle, il suffit que le débit de sel traversant la section de prélèvement soit égal au débit de sel injecté pendant la durée du régime permanent seulement.

Dans la méthode proposée, il est indispensable que tout le sel injecté passe à travers la section de prélèvement.

\section{II. - EXPÉRIMENTATION}

Les premiers jaugeages par la méthode proposée ont été entrepris par la Division Technique Générale à la fin de l'année 1959. Près de cinyuante essais ont été effectués depuis sur diverses rivières des Alpes, des Pyrénées et du Massif Central, la plupart de ces essais comprenant plusicurs mesures de vérification.

L'expérimentation a porté essentiellement sur la vérification directe de la première hypothèse fondamentale et sur la comparaison des résultats avec ceux des méthodes traditionnelles (moulinet, méthode chimique).

\section{II-1. - Vérification de la première condition fondamentale.}

Le fait qu'une rivière puisse avoir assez de sollicitude à l'égard de l'hydrologue pour répartir le sel de façon que la première hypothèse fondamentale $\int_{0}^{\mathrm{I}} c_{2} d t=\mathrm{C}^{\text {te }}$ soit réalisée, peut apparaître comme l'affirmation d'un postulat irréalisable, qui semble d'ailleurs avoir été ignoré par les promoteurs de la méthode par intégration.

Cette condition a pourtant été vérifiée sur plusieurs torrents ou rivières des Alpes, des Pyrénées et du Massif Central avec des écarts inférieurs à $5 \%$ entre les valeurs de $\int_{0}^{T^{\prime}} c_{2} d t$ obtenues pour des échantillons prélevés en divers points d'une même section ou dans des sections différentes.

Dans une même section, les points de prélèvements étaient répartis entre les zones de fortes vitesses et de faibles vitesses, près des berges et au centre, à diverses profondeurs. Dans une même rivière, plusieurs séries de prélèvements ont été faites en des sections assez éloignées pour que les temps de passage y soient dans le rapport de un à deux. En général, deux ou trois points de prélèvements étaient retenus pour chaque essai.
Le tableau ci-dessous donne les résultats de ces essais faits lors des premières mesures effectuées par ce procédé.

\begin{tabular}{|c|c|c|c|}
\hline RIVIÈRE & LIEU & $\begin{array}{l}\mathrm{DEE} B \mathrm{~T} \\
\mathrm{~m}^{3} / \mathrm{s}\end{array}$ & 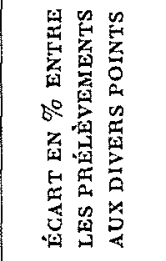 \\
\hline Ferrand. . . & Mizoen. ..... & 0,500 & 1,1 \\
\hline Merderel . . . & Saint-Martin . & 0,100 & 4 \\
\hline Bugeon ...... & Saint-Francois. & 0,170 & 3 \\
\hline Issole $\ldots \ldots$ & Saint-André. . . & 4,60 & 4,2 \\
\hline La Lyonne.. & Sources.... . & 0,500 & 2,5 \\
\hline Romanche... & Chambon... & 1,70 & 2,6 \\
\hline Durance.... & Vachette. ... & 2,34 & 0,4 \\
\hline Issole . . . . . & Saint-André. . & 2,50 & 2,5 \\
\hline Gravelles... & Courchevel.. & $\mathrm{X}$ & $\begin{array}{l}\text { résultats } \\
\text { inexpoi- } \\
\text { tables } \\
\text { bsorption } \\
\text { par la } \\
\text { neige }\end{array}$ \\
\hline Cerveyrette. . & Cervières ... & 1,6 & 4,3 \\
\hline Cristillan & La Viste.... & 5,62 & 0 \\
\hline La Blanche. . & La Garde... & 1,95 & 1,5 \\
\hline Ferrand .... & Mizoen .... & 9,7 & 1,5 \\
\hline Cerveyrette. . & Cervières... & 9,10 & 0,5 \\
\hline
\end{tabular}




\section{1-2. - Vérification de la deuxième hypothèse fondamentale dans le cas du bichromate.}

\section{II-2.1. RÉDUCTION DU BICHROMATE.}

Une longue expérience de la méthode de dilulion traditionnelle portant sur près de 2000 jaugeages exécutés à des stations où sont également réalisées des mesures au moulinet, permet de penser que les cas de réduction chimique (1) du bichromate par des eaux des torrents de montagne sont très rares. Les écarts entre les jaugeages chimiques et les mesures au moulinet effectués simultanément dans de bonnes conditions ne dépassent pas $3 \%$. Il faut toutefois noter que ces écarts sont presque tous de même sens, la méthode chimique conduisant à des débits plus élevés que les moulinets. Ceci peut être attribué à une faible disparition du bichromate aussi bien qu'à un freinage des moulinets en régime turbulent ou à la prise en compte d'une partie du débit sous-fluvial par la méthode chimique.

\section{II-2.2 Adsorption dU BICHRomate.}

D'autres phénomènes physiques peuvent conduire à ne pas retrouver dans l'eau de la section de mesure tout le bichromate injecté.

II-2.21 Adsorption par la berge : Cette adsorption est certaine lorsque les berges sont recouvertes de neige; on voit alors la berge se colorer. Elle est probable dans les zones herbeuses ou dans celles qui ne sont recouvertes par l'eau que de façon sporadique. II est vraisemblable que cetle adsorption n'est que temporaire et que les berges restituent lentement le bichromate à la rivière.

II-2.22 Adsorption par les matières en suspension : Ce phénomène a été constaté pour certaines eaux glaciaires transitant de fines particules en suspension. Si on filtre les échantillons ou si on les laisse longuement décanter, on constate par la méthode colorimétrique, après adjonction du réactif, que l'eau claire contient moins de bichromate que l'eau trouble.

Il suffit d'agiter vivement les échantillons pour que cette adsorption ne fausse pas l'analyse. Lors-

(1) La «réduction» du bichromate se traduit par la transformation des ions $\mathrm{Cr}^{+1++++}$ en ions $\mathrm{Cr}^{++++}$qui ne sont pas décelables à l'analyse colorimétrique avec le réactif utilisè. que la turbidité introduit des dispersions trop grandes, les échantillons sont agités, puis dilués avec de l'eau claire dans une proportion parfaitement connue, avant passage dans le colorimètre.

\section{II-3. - Comparaisons avec d'autres méthodes.}

Les erreurs résultantes dues à ces diverses causes ont été mises en évidence en comparant les résultats obtenus par la méthode par intégration proposée avec des jaugeages simultanés au moulinet, par la méthode traditionnelle ou simplement à l'aide de stations de jaugeage tarées par ces deux méthodes. Les écarts constatés sont portés dans les tableaux ci-après (on trouvera parfois deux écarts pour la même rivière, ils correspondent à des prélèvements effectués en des points différents) :

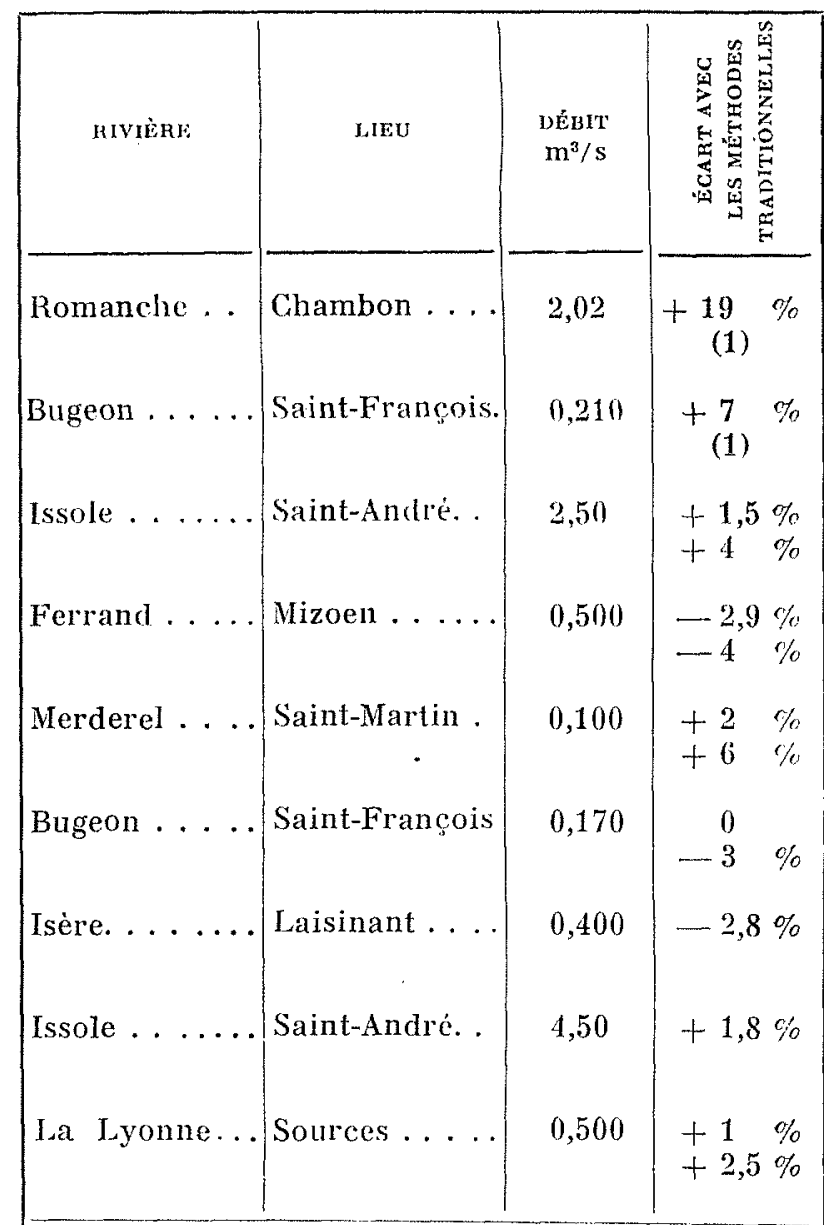

(1) Les prélèvements n'ont pas été poursuivis pendant un temps assez long; les derniers contenaient encore du bichromate. 


\begin{tabular}{|c|c|c|c|}
\hline RIVIẼRE & LIEU & $\begin{array}{l}D E ́ B I T \\
\mathrm{~m}^{3} / \mathrm{s}\end{array}$ & 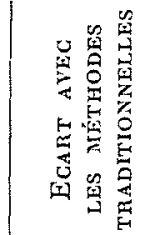 \\
\hline Romanche. . & Chambon ... & 1,6 & $\begin{array}{l}0 \\
-0,6 \\
+4\end{array}$ \\
\hline Durance .... & Vachette... . & 2,3 & $\begin{array}{l}-2,6 \\
-3\end{array}$ \\
\hline Gravelles... & Courchevel .. & & $\begin{array}{l}\quad(2) \\
\text { résultats } \\
\text { inexplai- } \\
\text { tables }\end{array}$ \\
\hline Cerveyrette. . & Cervières. . . & 1,64 & $\begin{array}{l}+0,7 \\
+5\end{array}$ \\
\hline Romanche. . & Chambon .... & 36,6 & 0 \\
\hline Cristillan ... & I. a Viste...... & 5,62 & 1,5 \\
\hline Le Guil....... & Chât.-Queyras. & 36,2 & $+0,5$ \\
\hline I a Blanche... & La Garde.... & 1,95 & $\begin{array}{l}0 \\
+1,5\end{array}$ \\
\hline Ferrand..... & Mizoen $\ldots$. & 9,7 & 0,7 \\
\hline \multicolumn{4}{|c|}{$\begin{array}{l}\text { (2) Dans ce cas, rétention importante par la neige : } \\
\text { la faiblesse du débit aurait nécessité plusieurs heures } \\
\text { de prélèvement pour la récupération de tout le } \\
\text { hichromate. }\end{array}$} \\
\hline
\end{tabular}

\begin{tabular}{|c|c|c|c|}
\hline nIVIíRE & MLEU & $\begin{array}{l}\text { D)́вит } \\
\mathrm{m}^{3} / \mathrm{s}\end{array}$ & 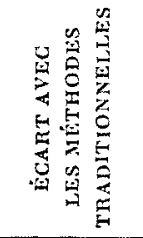 \\
\hline Articol, . & Articol & 0,270 & $+3.7 \%$ \\
\hline Ferrand .... & Mizoen . . . . . & 2,33 & $-0,4 \%$ \\
\hline La Blanche.. & La Garde... & 1,92 & 0 \\
\hline Isère .... & Laisinant . . . . & 1,52 & 0 \\
\hline Romanche . . & Chambon ... & 7,30 & 0 \\
\hline Ferrand ... & Mizoen ...... & 13,0 & $-3,8 \%$ \\
\hline Lyonne. . . & Bouvante... & 1,22 & () \\
\hline Trélatête... & La Laya..... & 0,260 & 0 \\
\hline Dorinet . . . . & Belleville . . . & 0,684 & $-6 \%$ \\
\hline Isère. & Laisinant .... & 0,770 & 0 \\
\hline
\end{tabular}

\section{III. - MODE OPERATOIRE}

\section{III-1. - Sur le terrain.}

\section{III-1.1. INJECTION.}

Si la rivière est peu connue des opérateurs, il est préférable de prévoir un essai d'injection instantanée de fluorescéine pour déterminer les sections d'injection et de prélèvement, les temps de propagation et d'étalement du nuage. En fait, l'examen à l'œil ne permet pas de déceler les traces de fluorescéine à la fin du passage et il est bon de considérer que le nuage salin passe au lieu de prélèvement pendant un temps $T$ double de celui évalué à l'œil.

Lorsque la rivière est bien connue des opérateurs, l'essai préalable à la fluorescéine n'est pas indispensable. La fluorescéine peut être, le cas échéant, déversée simultanément à l'injec- lion de bichromate: l'analyse colorimétrique n'est pas faussée, ou très peu (moins de $1 \%$ ), les colorations habituelles en fluoresceine étant peu visibles pour les épaisseurs, voisines de $1 \mathrm{~cm}$, des échantillons.

La quantité de solution de bichromate à déverser est fonction du temps de passage $\mathrm{T}$ et $\mathrm{du}$ débit $Q$ de la rivière (que l'on évalue grossièrement) : le volume à ensemencer est en effet égal à $Q \times \mathrm{T}$. On injecte un poids minimum de bichromate $\mathrm{P}$ suffisant pour que la concentration moyenne $P /(Q \times T)=C_{2}$ soit facilement dosable par la méthode d'analyse, soit en général 0,2 à $1,5 \mathrm{mg} / \mathrm{l}$ pour la méthode colorimétrique.

Le bichromate est transporté le plus souvent en solution concentrée à $600 \mathrm{~g} / 1$ environ, dans des bonbonnes en matière plastique de capacités connues, échelonnées de 0,5 litre à 20 litres. 
Une mêne solution, préparée dans des cuves de 500 litres, peut être utilisée pour de nombreux jaugeages, ce qui procure un gain de temps très appréciable à l'analyse (voir III-2.4) lorsqu'une précision de 2 à $3 \%$ est suffisante.

Cette méthode nécessite le transport inutile d'eau. Aussi, dans le cas de longues marches en montagne, le bichromate est-il transporté en paillettes dans les mêmes bonbonnes. L'eau est ajoutée sur le lieu de jaugeage. Il est alors nécessaire d'effectuer un prélèvement de cette solution mère avant l'injection.

Le déversement de la solution se fait de facon quelconque en rinçant soigneusement les récipients pour que tout le volume de solution soit déversé dans la rivière. II y a lieu de ne pas verser trop brutalement cette solution pour éviter une concentration trop forte de la rivière au point d'injection.

Les concentrations maximales observées au lieu de prélèvement ne dépassent jamais $3,5 \mathrm{mg} / \mathrm{I}$, concentration qui ne présente aucun danger.

\section{III-1.2 PRÉLİVEMENTS.}

On verra plus loin (III-2.2) que le nombre minimal d'échantillons à prélever pour avoir une précision satisfaisante est indépendant du teurps de passage du nuage au lieu de prélèvement.

Un total de 25 prélèvements permet d'obtenir une précision satisfaisante, compatible avec les conditions de jaugeage. Les échantillons sont pris à des intervalles de temps égaux : on définit cet intervalle de façon que le premier de ces échantillons, ainsi que les deux ou trois derniers, ne contiennent pas de bichromate.

Les échantillons sont tous prélevés en un même point de la section, dans des flacons en verre ou en matière plastique d'un volume de $50 \mathrm{~cm}^{3}$, parfaitement propres et secs, soit au moyen de godets de prélèvements, soit de préférence à la main, si possible dans la zone de plus fort conrant. Les heures de prélèvements sont définies soit à l'aide d'un chronomètre, soit de préférence à l'aide d'une montre à trotteuse centrale.

On peut envisager la réalisation sur le terrain d'un ou plusieurs échantillons moyens :

Un premier procédé consiste à prélever un débit constant pendant toute la durée du passage du nuage salin, au moyen d'une pompe portative : ce procédé fait perdre à la méthode par intégration une partie de son intérêt si le matériel de prélèvement est trop lourd; aussi l'utilisation d'une micro-pompe de laboratoire d'un poids de quelques centaines de grammes, d'un débit voisin de 10 litres par heure et alimentée par piles sèches, est-elle à l'étude.

Il est possible de constituer aisément un échantillon moyen en donnant à tous les prélèvements des volumes égaux. Le mélange de tous ces prélèvements est fait au fur et à mesure de leur prise avec un même récipient.

Après une période d'essais, nous utilisons généralement cette dernière méthode.

Dans certains cas, on réalise trois ou quatre échantillons moyens de 0,5 à 1 litre composés de nombreux prélèvements de $5 \mathrm{~cm}^{3}$ effectués pendant tout le temps de passage, en trois ou quatre points.

Dans d'autres cas, trois ou quatre échantillons moyens sont prélevés pendant des temps différents (voir III-2.3), le premier prélèvement de chaque échantillon étant toujours effectué au temps origine.

\section{III-2. - Analyse colorimétrique au laboratoire.}

Le principe de l'analyse est le même que dans la méthode traditionnelle : on compare les échanlillons prélevés à des dilutions connues de la solution injectée, réalisées avec l'eau naturelle de la rivière. Cette comparaison est faite à l'aide de colorimètres à cellules photoélectriques.

Plusieurs méthodes peuvent être utilisées pour le dépouillement.

III-2.1 ANALYSE JJE TOUS LES ÉChaNTHLlons.

Dans ce cas, on compare chacun des échantillons prélevés avec les dilutions de la solution injectée.

La comparaison colorimétrique permet de définir pour chaque échantillon la valeur du rapport $c_{2} / \mathrm{C}_{1}$ de la concentration de l'échantillon à la concentration de la solution injectée. Les valeurs de ce rapport sont portées sur un graphique en fonction du temps (fig. 1).

Le planimétrage de la surface $S$ permet de dćfinir la valeur de l'expression :

$$
\mathrm{S}=\left(1 / \mathrm{C}_{1}\right) \int_{0}^{\mathrm{T}} c_{2} d t
$$

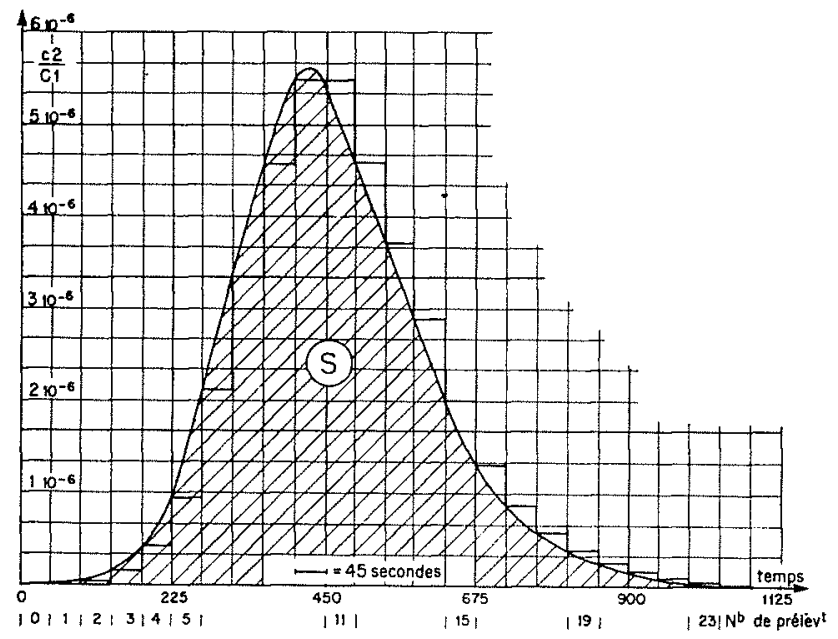

FIG. 1

Le Crislillan a Lal Viste 
donc le débit défini par la formule (1):

$$
\mathrm{Q}=\frac{\mathrm{VC}_{1}}{\int_{0}^{\mathrm{T}} c_{2} d t}
$$

\section{Inconvénient de cette méthode.}

Ce mode de dépouillement est long car il nécessite la préparation d'une dizaine de dilutions échelonnées de $10^{-5}$ à $5 \cdot 10^{-7}$ et l'analyse de chacun des échantillons prélevés.

par ailleurs, la sensibilité du colorimètre est mauvaise pour les derniers échantillons très peu concentrés : la partie décroissante de la courbe et son point de contact avec l'axe des temps sont donc mal définies.

\section{III-2.2 Anal.yse D’un Échantilloon MOYen.}

On mélange des quantités égales de tous les échantillons prélevés en un même point dans le temps $\mathrm{T}$ et on compare l'échantillon moyen ainsi constitué aux dilutions connues de la solution injectée.

Cette méthode présente l'avantage d'être très rapide et d'offrir à l'analyse un échantilion moyen dont la concentration a été calculće pour correspondre à la zone de sensibilité optimum du colorimètre $(0,2$ à $1,5 \mathrm{mg}$ par litre en général).

\section{Inconvénient :}

Un seul échantillon est analysé : on augmente ainsi les risques d'erreurs; il suffit d'un flacon mal lavé ou d'une erreur dans la préparation de l'échantillon moyen pour entraîner une erreur importante sur la valeur du débit mesuré.
Cet inconvénient n'existe pas si plusieurs séries de prélèvement sont faites en divers points de la rivière, mais le nombre total de prélèvements devient vite excessif.

Par ailleurs, le fait de mélanger des volumes égaux de plusieurs échantillons prélevés à des intervalles de temps constant revient à faire une moyenne et non une sommation et conduit à une erreur par excès (fig. 1).

Une courte étude mathématique a conduit M. Mazeran, Ingénieur à la Division Technique Générale, à penser que l'erreur ainsi commise est voisine de $1 /(n+1)$, si $n$ représente le nombre d'échantillons contenant du bichromate.

Aussi, pour six courbes expérimentales de formes très diverses et correspondant à des temps de passage écheionnés de 500 à 1600 secondes, a-t-il comparé les écarts obtenus entre la sommation et la moyenne de $n$ échantillons avec les valeurs correspondantes de $1 /(n+1)$. Les résultats sont portés dans le tableau I.

Ce tableau met en évidence que les écarts constatés sont très voisins des valeurs de $1 /(n+1)$, ce qui conduit M. Mazeran à adopter le mode de dépouillement suivant :

1. L'échantillon moyen est composé d'un cehantillon blanc (eau naturelle sans bichromate) et de $n$ échantillons contenant du bichromate.

2. Le temps de passage est considéré comme étant égal à $(n+1) t$ et non à $n t$. On compense ainsi l'erreur systématique due au remplacement de la sommation par la moyenne.

Le tableau ci-dessus montre que l'on a intérêt à prendre au moins 25 échantillons pour que le terme correctif ne soit pas trop grand.

III-2.3 Une troisième méthode proposée par

TABLEAU I

\begin{tabular}{|c|c|c|c|c|c|c|}
\hline \multirow{3}{*}{ RIVIÈRE } & \multirow{3}{*}{ LIEU } & \multirow{3}{*}{$\begin{array}{l}\text { TEMPS } \\
\text { DE PASSAGE DU SEL } \\
\text { EN SECONIDS }\end{array}$} & \multicolumn{4}{|c|}{ NOMBRE D'ÉCHANTILLONS PAÉLEVÉS $n$} \\
\hline & & & $n=50$ & $n=\overline{25}$ & $n=15$ & $n=12$ \\
\hline & & & \multicolumn{4}{|c|}{ ÉCARTS ENTRE LA SOMMATION ET LA MOYENNE } \\
\hline Oriège ... & Pont de Bisp....... & $T=525$ & $+1,9$ & 3,5 & 6 & 7,1 \\
\hline Pipe-Line & (Amérique) . . . . & $\mathrm{T}=600$ & +2 & 3,8 & 6 & 7,9 \\
\hline Bugeon ....... & Saint-François . . . & $\mathrm{T}=900$ & $+1,6$ & 3,4 & 6 & 7,1 \\
\hline Pipe-Line . . . . . & (Amérique) & $\mathrm{T}=1000$ & $+2,2$ & 4,0 & 6,1 & 8,1 \\
\hline Issole ......... & Saint-André ..... & $\mathrm{T}=1150$ & $+1,8$ & 3,7 & 6,8 & 7,5 \\
\hline Pipe-Line . . . . . & (Amérique) . . . & $\mathrm{T}=1600$ & +2 & 3,9 & 6 & 7,7 \\
\hline \multicolumn{2}{|c|}{ Moyenne des écarts } & & 1,9 & 3,7 & 6,1 & 7,6 \\
\hline \multicolumn{2}{|c|}{ Valeur de $\frac{1}{n+1}$} & & 1,95 & 3,85 & 6,25 & 7,7 \\
\hline
\end{tabular}


M. Mazeran permet d'éliminer partiellement les inconvénients des deux premières :

On analyse un certain nombre d'échantillons moyens, quatre par exemple.

Le premier est un mélange à volumes égaux de un échantillon blanc et des $n-n_{1}$ premiers échantillons contenant du bichromate.

Le deuxième, de un échantillon blanc et des $n-n_{2}$ premiers échantillons.

Le troisième, de un échantillon blanc et des $n-n_{3}$ premiers échantillons, $n_{1} n_{2} n_{3}$ étant des nombres compris entre $n / 2$ et 1 , par exemple.

Le quatrième, de un échantillon blanc et des $n$ échantillons contenant du bichromate.

On calcule le débit d'après l'analyse de chacun des quatre échantillons moyens et on reporte les quatre débits ainsi obtenus sur un graphique (fig. 2) en fonction du nombre de prélèvements.

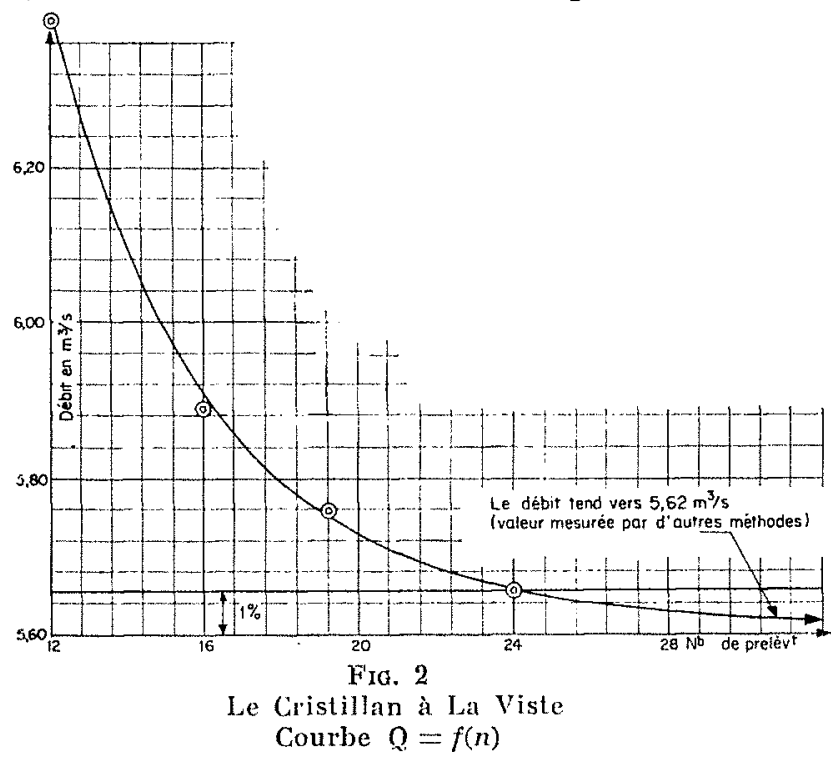

La courbe tracée à partix de ces quatre points lend vers une limite dont l'ordonnée est égale au débit : celte ordonnée est théoriquement égale à celle du quatrième point obtenu à partir de tous les échantillons contenant du bichromate.

Ce procédé présente plusieurs avantages :

a) Plusieurs échantillons sont analysés, il y a ainsi compensation partielle des erreurs;

b) Comme il est difficile de deceler avec certitude si les derniers échantillons comportent ou non du bichromate, le tracé de la courbe permet d'apprécier plus aisément si le quatrième échantillon moyen contient effectivement tout le bichromate qu'il devrait contenir;

c) Si les opérateurs sur le terrain n'ont pas prélevé suffisamment longtemps et si le dernier prélèvement contient encore du bichromate, il est possible, par cette méthode, de définir, par: extrapolation, une valeur approchée du débit. Dans ce cas, on peut multiplier le nombre d'échantillons moyens comprenant en particulier les $(n-5)$, puis $(n-4) \ldots(n-1)$ premiers echantillons.

Ce même procédé est utilisé lorsque les trois ou quatre échantillons moyens ont été réalisés sur le terrain par prélèvements de volumes cgaux.

\section{Inconvénients :}

L'extrapolation de la courbe $Q=f(n)$ est sentimentale et susceptible d'interprétations diverses.

Les possibilités d'un calcul plus cartésien sont en cours d'étude, mais il faut préciser que les risques d'erreurs sont faibles, car la plus grande partie du bichromate passe en un temps très

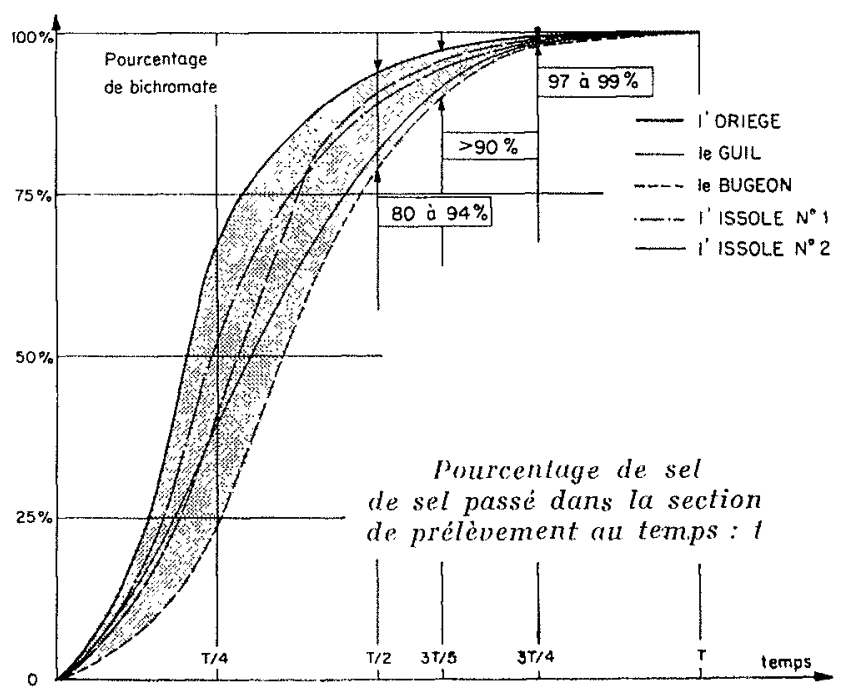

FIG. 3

Méthode chimique de dilution. Procédé par intégration.

court, ce qui est mis en évidence sur le graphique de la figure 3.

\section{III-2.4 DILUTION DE LA SOLUTION MÈRE.}

Quel que soit le mode de dépouillement choisi, il est nécessaire de comparer les échantillons prélevés à des dilutions, préparées en laboratoire, de la solution injectée.

$\mathrm{Si}$ on veut obtenir une excellente précision, il est indispensable de préparer ces dilutions pour chaque dépouillement à partir d'un échantillon de la solution mère, prélevé immédiatement avant injection et dilué avec l'eau naturelle de la rivière.

Si une précision de l'ordre de $3 \%$ est suffisante, on peut se contenter de faire une seule analyse colorimétrique des dilutions d'une même solution mère utilisée pour plusieurs jaugeages, ce qui permet d'obtenir «la courbe de réponse » du colorimètre à la solulion utilisée. 


\section{Ceci suppose :}

$1^{\circ}$ Que les réactifs préparés à des dates et avec des produits de provenances diverses produisent les mêmes colorations à concentrations égales;

2“ Que les solutions concentrées de bichromate sont stables dans le temps;

$3^{\circ}$ Que les dilutions de mème valeur obtenues avec les eaux des diverses rivières ont les mêmes densités optiques;

Cette troisième condition élimine la méthode proposée lorsque les eaux naturelles sont très troubles ou colorées (a fortiori lorsque leurs caractéristiques chimiques modifient l'action du réactif, mais dans ce cas la méthode de dilution au bichromate est inapplicable).

Les divers essais qui ont été faits dans les torrents de montagne des Alpes et des Pyrénées montrent que ce mode d'analyse simplifié entraîne le plus souvent des erreurs inférieures à $3 \%$.

Le dépouillement par ce procédé d'un jaugeage effectué sur le Cristillan en utilisant une solution de bichromate préparée et analysée au colorimètre plus de trois mois auparavant, a conduit à un écart inférieur à $1 \%$ sur le débit obtenu par la méthode traditionnelle.

Il est d'ailleurs toujours possible de contrôler la courbe de réponse du colorimètre en analysant périodiquement une dilution déterminée d'un prélèvement de la solution mère injectée.

\section{IV. - POSSIBILITÉS DE LA MÉTHODE PROPOSEE}

\section{IV-1. - Avantages.}

Les avantages principaux de cette méthode dans son application aux jaugeages des torrents sont la légèreté, la rapidité et l'économie. La photographie de la figure 4, qui représente le maté- riel nécessaire au jaugeage d'un débit de torrent de $5 \mathrm{~m}^{3} / \mathrm{s}$, illustre en particulier la légèreté du procédé. Encore faut-il souligner que le godet et la bonbonne de prélèvement d'eau de la rivière sont le plus souvent inutiles...

Le poids du bichromate nécessaire est d'envi-

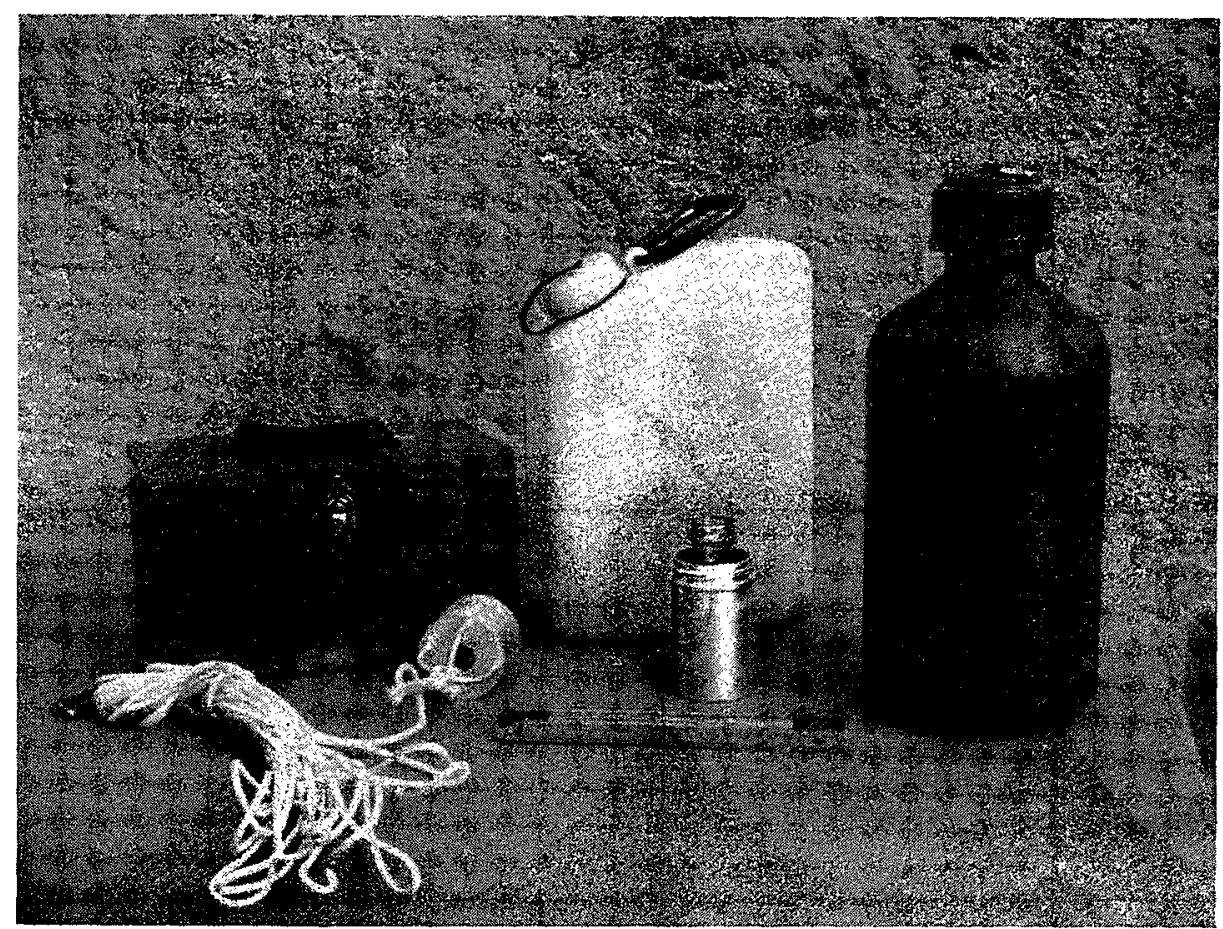

Fig. 4

Caissetle et godet de prélèvenents.

Bonbonnes pour solution concentré ef ea de la rivière. (Le mètre donne léchelle.) 
ron $500 \mathrm{~g}$ par $\mathrm{m}^{3} / \mathrm{s}$ jaugé dans les torrents, $1 \mathrm{~kg}$ pour $1 \mathrm{~m}^{3} / \mathrm{s}$ dans les rivières peu tourmentées, soit deux à trois fois moins que par la méthode de dilution traditionnelle.

Tous les hydrologues qui ont expérimenté par eux-mêmes l’ordre de grandeur du « gramme par homme et par mètre d'altitude », apprécieront cet avantage à sa juste valeur.

La réalisation d'un jaugeage, par une équipe de deux hommes éntraìnés connaissant la rivière, demande moins d'une demi-heure. Le jaugeage peut éventuellement être fait par un seul opérateur. La durée «active» du jaugeage est de l'ordre de 15 minutes, assez courte pour que le débit de la rivière n'évolue que très peu.

Si l'on veut obtenir une excellente précision, le dépouillement au laboratoire est fait dans le même temps que celui d'un jaugeage par la méthode chimique traditionnelle $(1 \mathrm{~h} 1 / 2)$. Il peut être fait en un temps trois fois plus court si on adnet que la courbe de réponse du colorimètre est tracée une seule fois pour tous les jaugeages faits avec une même solution.

Par ailleurs, le dépouillement peut être conduit sur le terrain, la courbe de réponse du colorimètre à la solution analysée ayant été établie en laboratoire : l'analyse colorimétrique des échantillons n'exige en effet pas de matériel fragile, alors que la réalisation des dilutions nécessaires à l'établissement de la courbe de réponse du colorimètre nécessite l'utilisation d'une abondante verrerie. On peut ainsi analyser les échantillons immédiatement après le jaugeage, ce qui réduit les risques de réduction du bichromate en solution diluée.

Enfin, signalons que le procédé par intégration convient parfaitement pour des analyses par résistivité, pour l'utilisation de radio-éléments tels que le brome, l'or, l'iode et le tritium.

\section{IV-2, - Inconvénients.}

Cette méthode présente plus de risques d'erreurs que la méthode traditionnelle; en effet : $1^{\circ}$ Chaque échantillon a une part de responsabilité dans le résultat final, alors que, dans la méthode traditionnelle, un échantillon $\mathrm{du}$ régime permanent peut être éliminé s'il est suspect;

$2^{\circ}$ La vérification de la qualité du brassage par la rivière est plus difficile, car elle exige le prélèvement de plusieurs séries d'échantillons;

$3^{\circ}$ L'adsorption de bichromate par les berges a une influence directe sur l'évaluation du débit et elle ne semble en particulier pas négligeable quand les berges sont recouvertes de neige.

\section{IV-3. - Possibilités d'utilisation.}

Les avantages et inconvénients précités, la comparaison des résultats obtenus dès les premiers jaugeages par la méthode proposée avec ceux des autres modes de mesure et mettant en évidence des écarts de signe quelconque toujours inférieurs à $5 \%$, souvent voisins de $2 \%$, conduisent à nous faire adopter peu à peu cette méthode pour la plupart des jaugeages de torrents.

Ces jaugeages nécessitent rarement une très grande précision, et nous estimons préférable de multiplier les mesures pour le tarage d'une station de jaugeages plutôt que d'obtenir une excellente précision sur une mesure isolée, ceci dans le cas où la méthode n'introduit pas d'erreurs systématiques, mais seulement une plus grande dispersion; une courbe de tarage n'est en effet qu'une moyenne obtenue à partir de plusieurs mesures : il faut insister sur le fait que souvent cette courbe n'est pas stable dans le temps et que de nombreux contrôles sont indispensables. Toute méthode rapide et de mise en œuvre facile nous a semblé à ce titre digne d'être développée.

Les améliorations que pourront lui apporter les différents utilisateurs conduiront peut être à utiliser prochainement cette méthode par intégration, même dans les cas où une excellente précision est indispensable.

\section{V. - BIBLIOGRAPHIE}

Dumas (H.). - La méthode chimiçue pour la mesure du débit des cours d'eau. La Houllle Blanche, oct.nov. 1952 et juin-juillet 1953 .

Dumas (H.). - Jaugeages de cours d'eau par la méthode chimique. International Association for Hydraulic Structures Research, troisième réunion, 5, 6 et 7 septembre 1943.

Luxo (A.) et Guntz. - Mesure du débit des oueds nordafricains par la méthode chimique au bichromate. La Honille Blanche, $\mathrm{n}^{\circ}$ spécinl $\mathrm{B}, 1954$.

Bonvin, Dumas et LiEvre. - Etude de la diffusion saline en régime permanent dans une conduite circulaire. Bertrand Irmaos Trav. Condessa de Rio 7, Lisbon.
Goguel. - Jangeage chimique continu du Guil. La Houille Blanche, mai 1946.

Soctété Hydrotechnique de France. - Code d'essais des installations hydrauliques, fascicule $\mathrm{n}^{\circ} 4,1952$.

André (H.): - L'amélioration des mesures de débits dans les aménagements hydroélectriques. La Houille Blanche, $\mathrm{n}^{\circ}$ spécial $\mathrm{B}$, décembre 1959 .

Huzr (D. E.). - The Total-Count Technique: A new principle in flow measurement. International Journal of Applied Radiation and Isotopes, 1958.

Hermant (C.). - Application de la mesure des débits par la méthode de dilution comparée à la détermination du rendement des turbines. La Houille 
Blanche, $\mathrm{n}^{\circ} 6$, novembre 1959.

Horibert Moser et Neumaier (Ferdinand). - L'emploi d'isotopes radioactifs dans l'hydrologie. Atomkern Energie, $\mathrm{n}^{\circ} 1$, janvier 1957.

Ege (J.F.) et Silverman (Leslie). -- Réactif colorimétrique stable pour le chrome. Analytical Chemistry, vol. $19, n^{\prime \prime} 9$, septembre 1947 .
Kolupalla (Prof. Stepanas). - Les jaugeages dans les ouvrages et les centrales hydroélectriques. Lat Honille Blanche, $\mathrm{n}^{\circ} 4$, juin 1960 .

Zrriln. - Modalités pour le titrage des solutions employées dans la méthode chimique pour la mesure des débits (nitrite de soude). La Houille Blanche, août-septembre 1953 .

\section{I S C USSION}

\section{Président : M. Remenieras}

II. le Président remercie M. Avnré, et souligne l'intérêt de la méthode qu'il a exposée; elle a été recommandée, lors de récents congrès : a) par les Russes, avec dosages par résistivimétrie, pour mesurer économiquement le rendement des turbines hydrauliques. b) par des ingénieurs des Etats-Unis qui l'appliquent surtout au jaugeage des rivières, en employant de préférence comme "marqueur» un radio-isotope de l'or.

il. Henmant indiçue que, dernièrement, le département Fssais d'Electricité de France a fait quelques essais a l'usine d'Argentat, sur la Dordogne, en vue de mesurer le rlébit turbiné par le groupe bulbe, débit de l'ordre de $100 \mathrm{~m}^{3} / \mathrm{s}$. Le système d'injection était placé ì l'entrée du groupe, et la section de prélèvement, où le mélange était suffisamment homogène, $2 \mathrm{~km}$ à l'aval. Dans ces conditions, la durée de l'injection nécessaire pour obtenir un régime permanent de concentration aurait été prohibitive; on a donc été amené à utiliser le même procédé que celui décrit par M. ANDrÉ. II fallait prélever des échantillons pendant tout le passage du nuage salin, ce qui durait environ $2 \mathrm{~h} 1 / 2$.

Ciette mélhorle de prélèvement a été combinée à unc méthode de relevés continus par résistivité. La solution concentíe injectée était en effet un mélange de chlorure de sodium et de bichromate de sodium, le bichromate étant analysé par voie colorimétrique et le chlorure étant ajouté pour produire dans la section de prélèvement, une variation de résistivité suffisamment importante pour être enregistrée.

Le même essai a été répété plusieurs fois et comparé avec le débit mesuré au moulinet.

Par analyse colorimétrique du bichromate, on a obtenu une dispersion entre les différentes mesures de l'ordre $1,4 \%$ et la valeur moyenne du débit ainsi mesuré était, par rapport aux moulinets, $6 \%$ par excès dans le cas de la méthode colorimétrique. Par contre, dans le cas des mesures de résistivité, la dispersion accidentelle des différents points est un peu plus grande, par suite des variations de la résistivilé naturelle, mais l'écart entre la valeur moyenne ainsi trouvée ei le débit déterminé par moulinet n'est plus que de $1,4 \%$ par excès.

En conclusion, dans le cas de cette application pratique à une usine hydraulique, le bichromate est mal adapté, et, il semble, en effet, que la seule explication soit une réduction du bichromate par les matières organiques que contieni l'eau de plaine. Cependant, dans son principe, la méthode paraît possible et mérite d'être poursuivie à l'aide de moyens appropriés.

M. le Président remercie M. Henmant pour ces intéressantes précisions.

M. Anpré, répondant aux questions de M. Plasse, indique que le volume $\mathrm{V}$ est obtenu à partir de bonbonnes de volumes différents compris entre 0,5 et 201 , jaugées d'avance au laboratoire. On en verse le nombre voulu dans la rivière pour obtenir la concentration désirée en estimant à vue le débit de la rivière.

M. Mamruth demande à M. ANDré quels sont les débits maxima qu'on avait pu jauger avec la méthode chimique traditionnelle, ainsi que les débits qu'on a mesurés avec la nouvelle méthode.

M. ANdré précise que la D.T.G. a jaugé par la méthode traditionnelle un débit voisin de $200 \mathrm{~m}^{3} / \mathrm{s}$. Aucune limite théorique n'est fixée, elle n'est fonction que de la qualité du brassage et du volume de la cuve d'injection.

Avec la nouvelle méthode, il n'a jamais eu l'occasion de mesurer plus de $50 \mathrm{~m}^{3} / \mathrm{s}$, mais cette méthode, exigeant denx ou trois fois moins de sel que la méthode traditionnelle, permet de jauger des débits plus forts.

M. Mamruth ajoute que, lors de son passage à la D.T.G. d'Electricité de France à Grenoble, il a eu l'occasion d'apprendre que la nouvelle méthode permettait de mesurer des débits triples des débits maximaux mesurés jusqu'à présent.

M. ANDRÉ répond que, théoriquement, on peut le faire, puisqu'il faut un volume de solution deux th trois fois plus faible. On risque toutefois d'ctre limité par le manque de brassage latéral dans les rivières $\dot{a}$ gros débits.

Yar ailleurs, on peut encore économiser du sel en injectant la quantité nécessaire pour un volume d'eau égal à $\mathrm{QT} / 2$ et non à $Q \mathrm{~T}$ ( $\mathrm{si}$ 《 $\mathrm{T}$ » est le temps de passage total du sel au lieu de prélèvement), et en faisant les prélèvements moyens pendant des temps variant entre $\mathrm{T} / 2$ et $\mathrm{T}$. Les deux derniers prélèvements sont peu concentrés et leur dosage imprécis; les deux premiers sont, au contraire, à une concentration convenable et conduisent déjà à une valeur approchée du débit (fig. 3). La courbe tracée à partir de ces quatro points permet une évaluation du débil avec une incertitude acceptable.

M. Anpré signale que le jaugenge d'un débit de $35 \mathrm{~m} / \mathrm{s}$ sur le Guil a été réalisé ainsi avec seulement $7 \mathrm{~kg}$ de bichromate; mais il s'agit sans doute d'un cas limite.

M. le Président invite M. Dimas, lun des promoteurs de la méthode elassique, à faire quelques commentaires sur ce. sujet.

Au nom du Laboratoire de Mécanique des Fluides de l'Université de Grenoble, innovateur de l'emploi du bichromate dans les jaugeages de rivières, M. Dumas cxprime sa satisfaction de voir que ce sel conserve toujours la faveur des expérimentateurs. Il se félicite de constater qu'après les réductions importantes de matériel obtenues dans la méthode traditionnelle par l'emploi de ce sel, la nouvelle technique exposée tend a une simplification cncore plus poussée des opérations sur le terrain. Les dernières incertitudes de la nouvelle teclnnique laissent encore au bon sens le soin de guider le choix entre les deux méthodes, selon les conditions locales et les disponibilités en moyens de transport et main-d'wuvre.

M. Dumas remercie M. André d'avcir exposé de luimême avec une grande riguelur toutes les objections qui pouvaient être opposées à la nouvelle technique.

M. le Président remercie M. Dumas de son intervention et M. André de son exposé. 